\title{
Auxetic shape memory alloy cellular structures for deployable satellite antennas: design, manufacture and testing
}

\author{
F. Scarpa ${ }^{1}$, S. Jacobs ${ }^{1}$, C. Coconnier ${ }^{1}, \mathrm{M} \mathrm{Toso}^{2}$, D DiMaio $^{1}$ \\ ${ }^{1}$ Department of Aerospace Engineering, University of Bristol, BS8 1TR Bristol, UK \\ ${ }^{2}$ ESA-ESTEC, Noordwijk, HOL
}

\begin{abstract}
We describe the production development and experimental tests related to an hybrid honeycomb-truss made of shape memory alloy (Ni48Ti46Cu6), and used as a demonstrator for a deployable antenna in deep-space missions. Specific emphasis is placed on the modal analysis techniques used to test the lightweight SMA structure..
\end{abstract}

\section{Background on SMA cellular structures}

Shape memory alloy (SMA) can be considered amongst the most successful smart materials to date in terms of spread of applications, and relative maturity of the technology. We do not aim in this work to provide an extensive review on the SMA field, however the interested reader can look for reference [1] for a detailed introduction on the subject. However, SMA honeycombs constitute a more recent development, being proposed by some of the Authors for the first time in 2004 at the SPIE Smart Materials and Structure Conference in San Diego, CA. Hassan, Scarpa and Mohammed [2] developed a OX (overexpanded) hexagonal honeycomb cold moulding some 1-way SMA ribbons, and gluing the hexagonal shapes obtained with a high temperature epoxy-based polymer. Almost at the same time, Shaw et al at the University of Michigan developed a patented assembling technique based on welding niobium rods, which allows producing small cellular samples with high manufacturing fidelity (i.e., low dimensional and precision tolerances) [3]. Prototypes of SMA honeycombs made with a similar welding techniques have been also manufactured and sandwich within composite face skins at the University of Tokyo. The sandwich panels subjected to low kinetic energy impacts and subsequent heating from martensite to austenite phase showed one of the main features for which SMA cellular structures have been developed - the capability of absorbing energy, and restoring (in a significant percentage) the original shape after impact [4]. Another interesting feature of SMA based honeycombs is also their intrinsic high hysteretic damping, 2-3 orders of magnitude higher than the commercial aluminium or steel-based honeycombs used in high end aerospace applications subjected to high cyclic fatigue loading or broadband vibration input [5].

The cellular structures considered so far have a centresymmetric topology - I.e., two perpendicular axis of symmetry. However, it is possible also to adopt a rotational-type of symmetry, which provides a combined in-plane rotation and translational deformation when subjected to inplane uniaxial loading, and feature also a negative Poisson's ratio behaviour. This structure, called hexagonal chiral topology, has been proposed as load-bearing concept by Prall and Lakes in 1996 [6], although the idea of negative Poisson's ratio in chiral-related structures was first devised by Wojciechowski for molecular assemblies [7]. The Poisson's ratio of this cellular structure is close to

This is an Open Access article distributed under the terms of the Creative Commons Attribution-Noncommercial License 3.0, which permits unrestricted use, distribution, and reproduction in any noncommercial medium, provided the original work is properly cited. 
-1 , approaching the limit of elasticity for isotropic materials. The ratio between the induced mechanical strains under pure thermal loading is also close to 1 [8], making this particular structure appealing in terms of folding-unfolding, and suggesting a possible use of the cellular concept for deployable antennas. This unusual deformation mechanism is the rationale behind the development of a chiral-based hybrid deployable honeycomb for satellite or probe antennas in deep space missions, where the deployable status is required as one-off, and the heating from the sun can provide the thermal loading required for the activation of the antenna.

\section{Manufacturing and development}

Hexachiral and Tetrachiral geometries are both chiral structures with negative Poisson ratio and hence are classed as auxetic materials (by Evans). They do not have a centre of symmetry and hence are defined as noncentrosymmetric or hemitropic by Prall and Lakes [6]. In both geometries, because axial stress is applied in plane to the structure, bending of the ligaments occurs and thus produces a rotation of the nodes. We can thereby pack the structure as small as the size of the nodes will let us. The ligaments roll around the nodes as shown hereafter in Figs. 1a and 1b. The node radius will limit the minimum packing size one can reach.

A rotation of the nodes will provoke the deformation of the honeycomb in $\mathrm{x}$ and $\mathrm{y}$ directions by an equal amount since $v_{\mathrm{xy}}=v_{\mathrm{yx}}=-1$. Prall and Lakes have reported that deformation due to axial compression and shear within the ligament itself can be ignored so long as the ligaments are suitably slender, thus keeping the relative density of the structure below a critical value [6]. It must be noticed that the rotational-translational deformation behaviour can be also achieved using another type of chiral tesselation (the so-called tetrachiral), where the unit cell if formed by 4-ligaments connectivity rather than the 6-ligament one in Figs. 1. The estimation of the in-plane stiffness of the tetrachiral and hexachiral configurations can be made using the formulas in [9]. In terms of antenna design, it is important to consider the weight to area ratio, one of the main performance drivers when considering that the cost of an average launch is around $10,000 \$$ per $\mathrm{kg}$ [10]. The weight calculations used for the carpet plots of Fig. 2 feature both hexachiral and tetrachiral structures the same constant node thickness of $7.5 \mathrm{~mm}$, ligament thickness of $0.25 \mathrm{~mm}$ and ligament width of $7 \mathrm{~mm}$, as used in a previous prototype [11]. It is clear from Fig.2 that tetrachiral honeycombs present lower and hence better weight to area ratios for a same node radius and ligament length than the hexachiral honeycomb. Generally we can say from these results that the tetrachiral structure is around 0.2 $\mathrm{kg} / \mathrm{m}^{\wedge} 2$ lighter than the hexachiral one that is around $\$ 2000 / \mathrm{m}^{\wedge} 2$ which is substantial. However the difference between the two reduces with increasing ligament lengths.

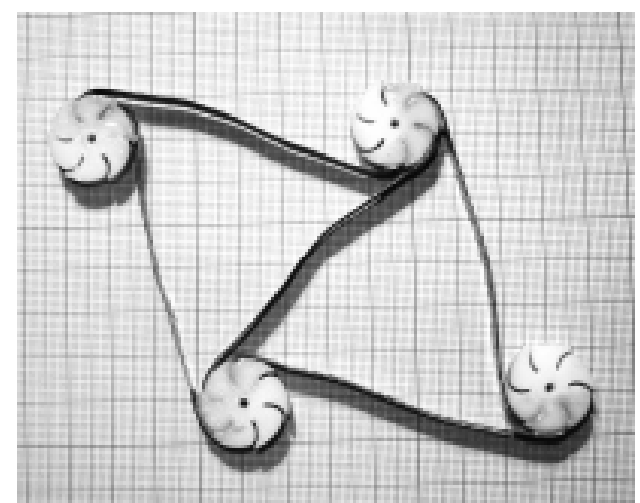

(a)

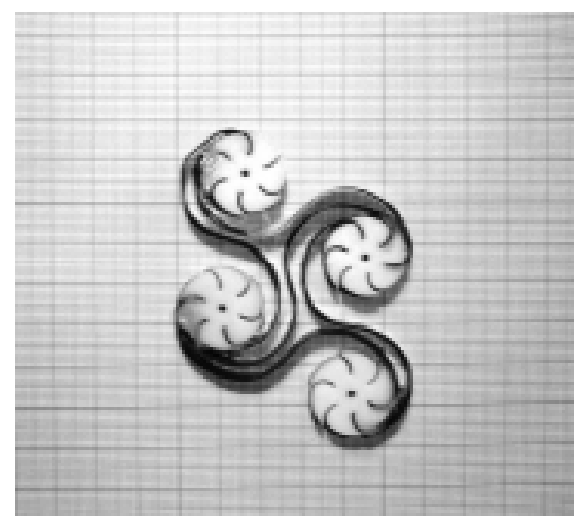

(b)

Fig 1. Extended (a) and compressed (b) hexagonal chiral unit cell 


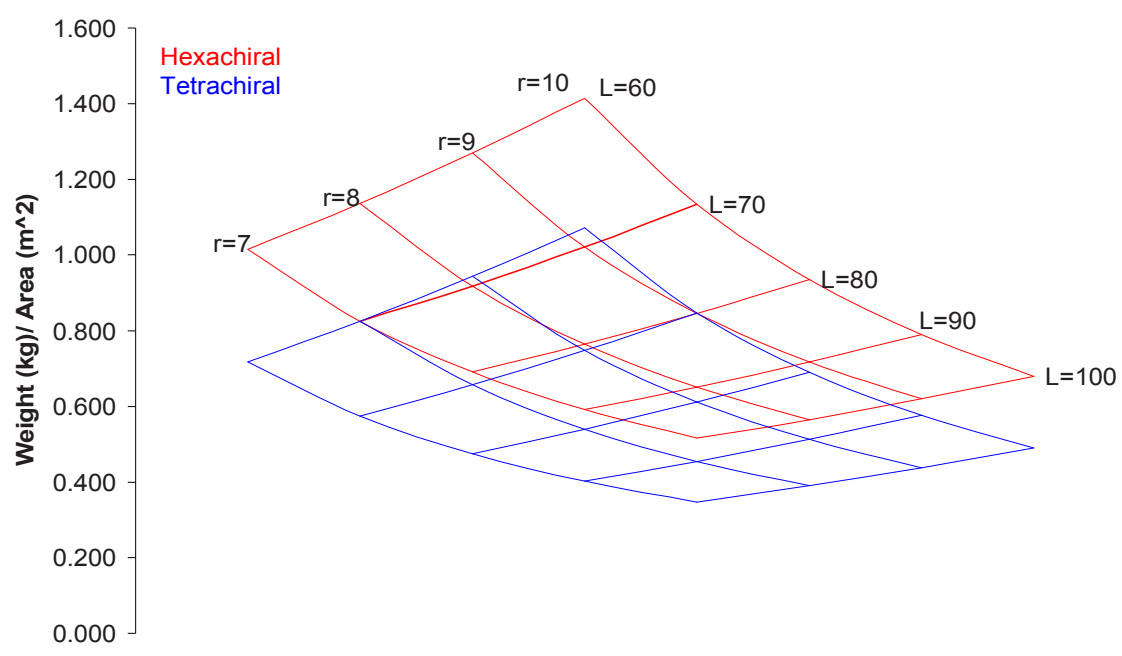

Fig. 2. Weight per Area ratio with varying $L$ and $r$

The objective of the chiral deployable antenna is that it can be packed into the launcher and then deployed to its full area once released in space. Hence it was apparent that the smaller the packed area to deployed area ratio had to be achieved. The packed to deployed area percentage of the two geometries was compared experimentally. It must be noted that the areas were measured from prototype structures with ligament length of $80 \mathrm{~mm}$ and node radius of $10 \mathrm{~mm}$ and the standard ligament thickness of $0.25 \mathrm{~mm}$. Hence results may lack precision for the un-forced packing ratio especially, since the honeycombs were packed mechanically by hand. The method used to do the unforced packing was to pack the honeycomb until the nodes were in contact with each other. The structure was then released from pressure and the area was measured by taking measurements from node centres. The forced packed area is the area of the honeycomb when the nodes are in contact with each other.

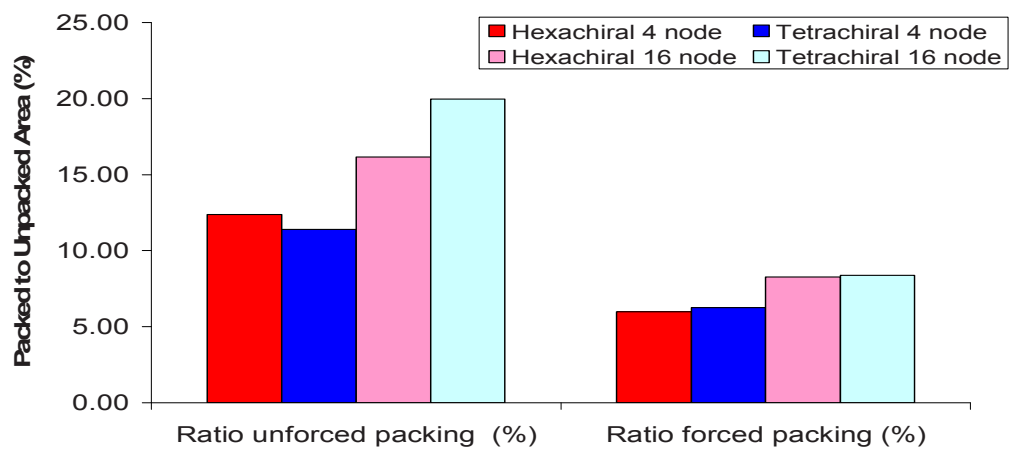

Fig. 3. Un-forced Packed to Forced Packed Area for 4 and 16 Node Honeycombs

The chiral unit cell needs a node specifically designed to accommodate the ligament, transmit the bending moment and, at the same time, guaranteeing the structural integrity and the load bearing 
capability of the assembly. A custom connecting node where the SMA ligaments could be bonded was designed as per Fig. 4. The slots in the node were purposefully made quite narrow; $0.72 \mathrm{~mm}$ leaving only $0.235 \mathrm{~mm}$ either side of the ligament for resin. This was made so as to avoid having to apply a very viscous resin, reduce the amount of bonding polymer needed per node to keep the weight low. The slots are curved out so as to leave room for the ligament's thickness when the ligaments roll up around the node in the packed state. The curve in the slot was also made intentionally so as to avoid stress concentrations at the joint. The inner radius of the curved slot is made sufficiently tight, with a radius of $3 \mathrm{~mm}$, so as to make room for spring back of the ligament when it is folded into the slot during manufacture.

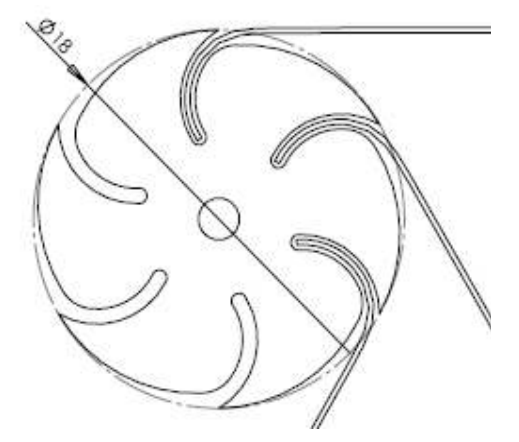

Fig. 4. Detail of Ligament to Node assembly

From a manufacturing and reliability perspective, the hexachiral configuration was preferred over the tetrachiral one because of the added degree of redundancy in the system, which is considered an essential feature for deep-space missions where no repairs on the structure are available. In producing real finite size samples, however, the part counts and dimensionality of the components has to be considered to predict the effective weight to mass ratio of the structure, as shown in Fig. 5.

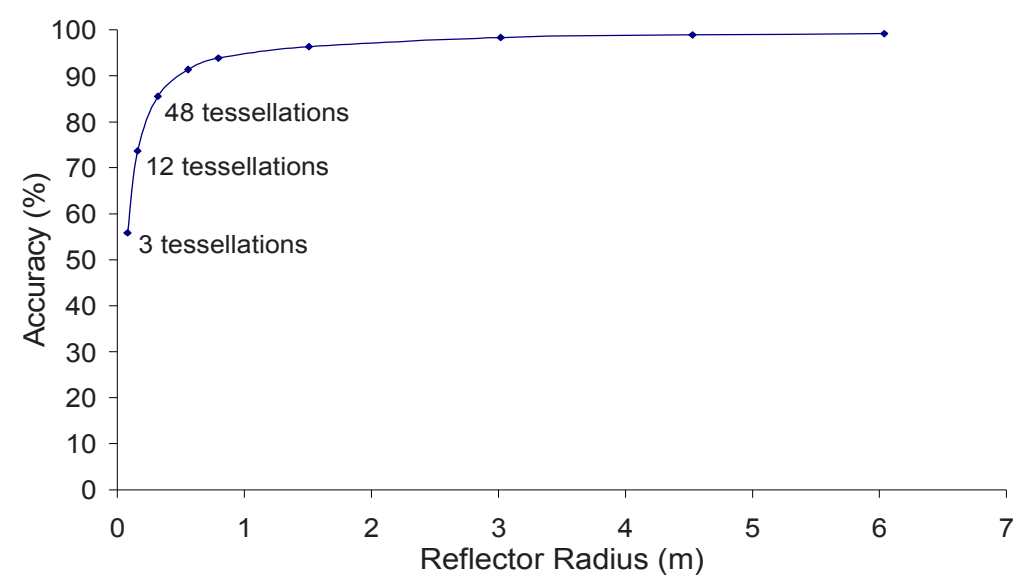

Fig. 5. Accuracy of Approximation for increasing number of tessellations

To support the analytical formulas used during the design stage, several FE multiphysics simulations involving in-plane uniaxial tensile loading of chiral structures have been performed, 
using models based on the ones described in [8]. The final chosen dimension for the prototype model has an aspect ratio of 10 and a relative density of 0.028 . The ligament length was chosen as $90 \mathrm{~mm}$ and the node radius chosen as $9 \mathrm{~mm}$ to correspond to the nondimensional aspect ratio and relative density simulated. These dimensions give an in-plane Young's modulus ratio of $3.8 \mathrm{MPa}$ and a packed to deployed percentage of $10.7 \%$, thus a deployment ratio close to $1: 10$. The weight to area ratio is 0.697 though it must be underlined that this value is for the un-equipped bare honeycomb structure of the reflector. Table 1 provides a summary of the materials selected for the final production of the antenna demonstrator.

Table 1. Chosen Materials and their key properties

\begin{tabular}{|c|c|c|c|c|c|}
\hline & Node & Ligament & \multicolumn{2}{|c|}{ Adhesive } & $\begin{array}{l}\text { Antenna } \\
\text { Surface }\end{array}$ \\
\hline Material & PEEK 1000 & NiTiNOL & $\begin{array}{l}\text { Redux } 810 \\
\text { (Prototype) }\end{array}$ & $\begin{array}{l}\text { Aremco 526N-C } \\
\text { (Space Structure) } \\
\end{array}$ & $\begin{array}{c}\text { Aluminised HN } \\
\text { Kapton }\end{array}$ \\
\hline CTE & 46.8 & 6.6 & $\mathrm{n} / \mathrm{a}$ & 32.4 & $\mathrm{n} / \mathrm{a}$ \\
\hline $\begin{array}{c}\text { Max Service Temp } \\
\left({ }^{\circ} \mathrm{C}\right)\end{array}$ & $300^{\circ} \mathrm{C}$ & $1200^{\circ} \mathrm{C}$ & $100^{\circ} \mathrm{C}$ & $300^{\circ} \mathrm{C}$ & \\
\hline $\begin{array}{c}\text { Min Service Temp } \\
\left({ }^{\circ} \mathrm{C}\right)\end{array}$ & $-120^{\circ} \mathrm{C}$ & & $\mathrm{n} / \mathrm{a}$ & $-60^{\circ} \mathrm{C}$ & \\
\hline$\rho\left(\mathrm{kg} / \mathrm{m}^{3}\right)$ & 1310 & 6450 & 1110 & 1230 & \\
\hline UV Resistance & Very Good & Very Good & $\mathrm{n} / \mathrm{a}$ & Good & \\
\hline Outgassing & Approved & Approved & $\mathrm{n} / \mathrm{a}$ & Acceptable & \\
\hline Special Features & Machinable & SME & $50^{\circ} \mathrm{C}$ Cure & & $\begin{array}{c}\text { Different } \\
\text { Coatings } \\
\text { available for } \\
\text { varying } \\
\text { wavelengths. }\end{array}$ \\
\hline
\end{tabular}

\section{Modal analysis of the antenna}

The antenna was subjected to a modal analysis test. The centrepiece of the SMA antenna was connected to an electrodynamics shaker (max static force $100 \mathrm{~N}$ ) and broadband white-noise excitation between $2 \mathrm{~Hz}$ and $25 \mathrm{~Hz}$ (Figure 6a). The loading was controlled through a Siglab/MATLAB system, while the structural velocities were acquired through a PSV-300F Polytec Scanning Laser Vibrometer. The single FRFs were measured in specific points marked with reflective tape the increase the coherence of the scattered laser beam. Each FRF was obtained through 20 averages and $50 \%$ overlaps, with a signal quality check option to accept only the measurements with higher correlation. The extraction of the mode shapes has been performed using the ICATS software (Imperial College, 2007). Modal complexity plots have been derived, as well as the values of the natural frequencies and modal loss factors (Figure $7 b$ ).

The natural frequencies extracted from the modal analysis technique were $8.14 \mathrm{~Hz}, 11.6 \mathrm{~Hz}, 15.2 \mathrm{~Hz}$ and $23.8 \mathrm{~Hz}$. The modal loss factors associated were $7 \%, 4.4 \%, 4.2 \%$ and $4.0 \%$ respectively. The values of the modal loss factors are quite significant, and two possible explanations can be found to justify the results. The first is related to the air damping contribution, which becomes sizeable for lightweight structures with high surface area. The second can be attributed to the intrinsic high loss factors of Nickel-Titanium-Copper ribbons, which at room temperature has been measured around 3 $\%$ [5]. The specific connection ligament-radius can originate also some dissipation mechanism, although it is likely to provide higher damping under in-plane torsion modes rather than out-of-plane deformations. For these lightweight structures, it is important also to notice the effect that the boundary conditions provide to the final experimental results. After several trials, the best fixture identified for the test was the fixing of the antenna to the rigid frame using a series of elastic bands, which however created an in-plane membrane tensional state. The numerical modal analysis carried 
out on an equivalent hexagonal shell Finite Element model approximating the antenna shows that the fundamental free-free mode should be an out-of-plane bending ("umbrella"), rather than a torsional one as in Figure 6b. On the opposite, the out-of-plane torsion mode should be the second one, always according to the FE model. Considering the effective isotropic mechanical response of the antenna structure, it is reasonable to argue that the membrane state created by the elastic bands provides a mode inversion for the system. It must be also noticed, however, that all the modes measured were above the $2 \mathrm{~Hz}-5 \mathrm{~Hz}$ threshold specified as minimum frequency ranges for acceptability in space antenna designs [10].

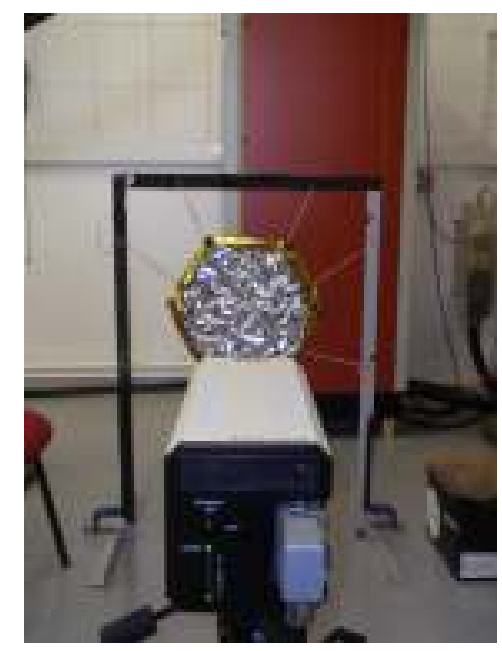

Fig. 6a. Modal analysis setup for the chiral antenna

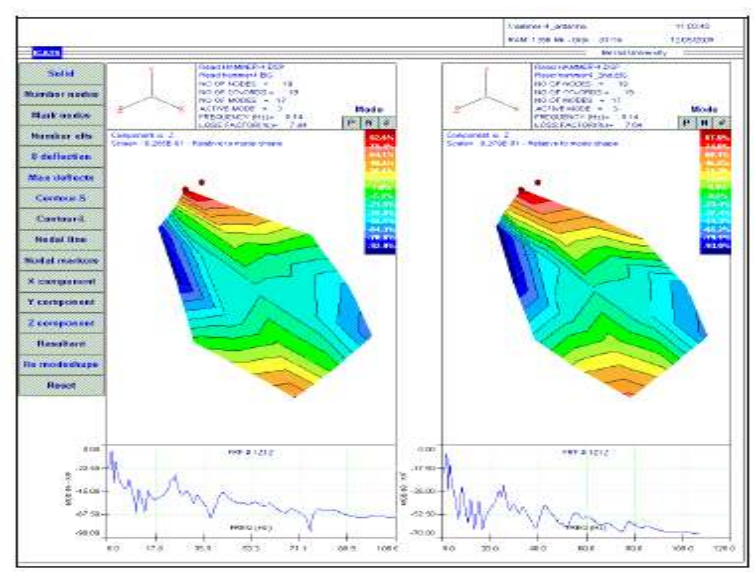

Fig. 6b. Complexity plots for the mode at $8.14 \mathrm{~Hz}$

\section{Conclusions}

We have described the design, manufacturing and testing of a deployable SMA antenna having a layout inspired to an auxetic (negative Poisson's ratio) structure. The antenna is made using a hybrid truss/honeycomb concept, where the ligaments provide axial deformation, and bending is transmitted through rotating cylinders. The modal analysis shows that the minimum thresholds for the modal response of the antenna satisfy the deep-space design requirements.

\section{Acknowledgements}

This work has been partially supported by the STRP-01364 FP6 CHISMACOMB project. Special thanks go to Mr J Martinez for assisting during the modal tests.

\section{References}

1. T W Duerig, Engineering Aspects of Shape Memory Alloys, Butterworth-Heinemann (1990)

2. M R Hassan, F Scarpa, N A Mohammed, Proceedings of SPIE, 5327:557-564 (2004)

3. J A Shaw, D S Grummon, J Foltz, Smart Materials and Structures, 16, 170-178 (2007) 
4. Y Okabe, S Minakuchi, N Shiraishi, K Murakami, Takeda N, Adv. Comp. Mat. 17(1), 41-56 (2008)

5. C Remillat, M H Hassan, F Scarpa, ASME J Engng. Mat. Tech., 128(3), 260-267 (2006)

6. D Prall, R S Lakes, Int. J. Mech. Sci., 39, 305-316 (1996)

7. K.W. Wojciechowski, Phys. Lett. A 137, 60-64 (1989)

8. M R Hassan, F Scarpa, N A Mohammed, M Ruzzene, Phys. Stat. Solidi B, 245(11), 2440-2444 (2008)

9. A Alderson, K L Alderson, D Attard, K E Evans, R Gatt, J N Grima, W Miller, N Ravirala, C W Smith, K Zied. Comp. Sci. Tech. DOI: 10.1016/j.compscitech.2009.07.009 (2009)

10. ESA-ESTEC, Private Communication. (2008)

11. M R Hasssan, F Scarpa, M Ruzzene, N A Mohammed, Mat. Sci. Engng. A, 481-482, 654-657 (2008) 